\title{
Engineering Spiny PtFePd@PtFe/Pt Core@multishell Nanowires with Enhanced Performance for Alcohols Electrooxidation
}
Yangping Zhang ${ }^{\dagger}$, Fei Gao $^{\dagger}$, Caiqin Wang* ${ }^{\ddagger}$, Yukihide Shiraishi ${ }^{\S}$, Yukou $D u^{* \dagger}$

${ }^{\dagger}$ College of Chemistry, Chemical Engineering and Materials Science, Soochow University, 199 Renai Road, Suzhou 215123, P.R. China

${ }^{\ddagger}$ College of Science, Nanjing Forestry University, 159 Longpan Road, Nanjing, 210037, P.R. China

$\S$ Tokyo University of Science Yamaguchi, Sanyo-Onoda-shi, Yamaguchi 756-0884, Japan

* Corresponding author:

* Yukou Du, E-mail: duyk @suda.edu.cn.

* Caiqin Wang, E-mail: wangcaiqin@njfu.edu.cn

(This document contains 13 pages, 2 tables and 18 Figures) 


\section{Calculation of ECSA:}

The electrochemical active surface area (ECSA) is one of the key factors estimating catalytic property towards electrooxidation of alcohols, which could be measured by an equation:

$$
\mathrm{ECSA}=\frac{\mathrm{Q}}{0.21 * \mathrm{~m}}
$$

Where Q can be calculated by integrating the area under the CV reduction peak, 0.21 $\mathrm{mC} \mathrm{cm}-2$ is a constant regarding the monolayer adsorption of hydrogen on Pt. The $\mathrm{m}$ represents the mass of Pt loading on the surface of GCE.

\section{Supporting Figures and Tables}

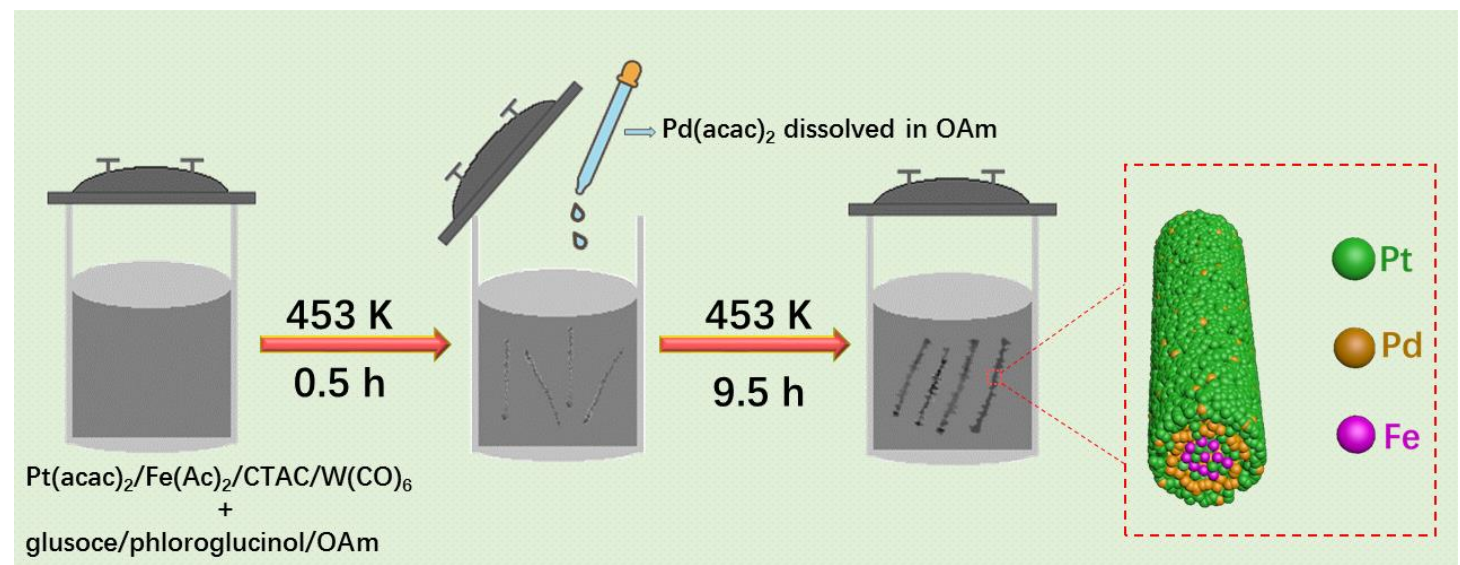

Figure S1. Schematic diagram of synthesis of PtFePd@PtFe/Pt core@multishell nanowires. 


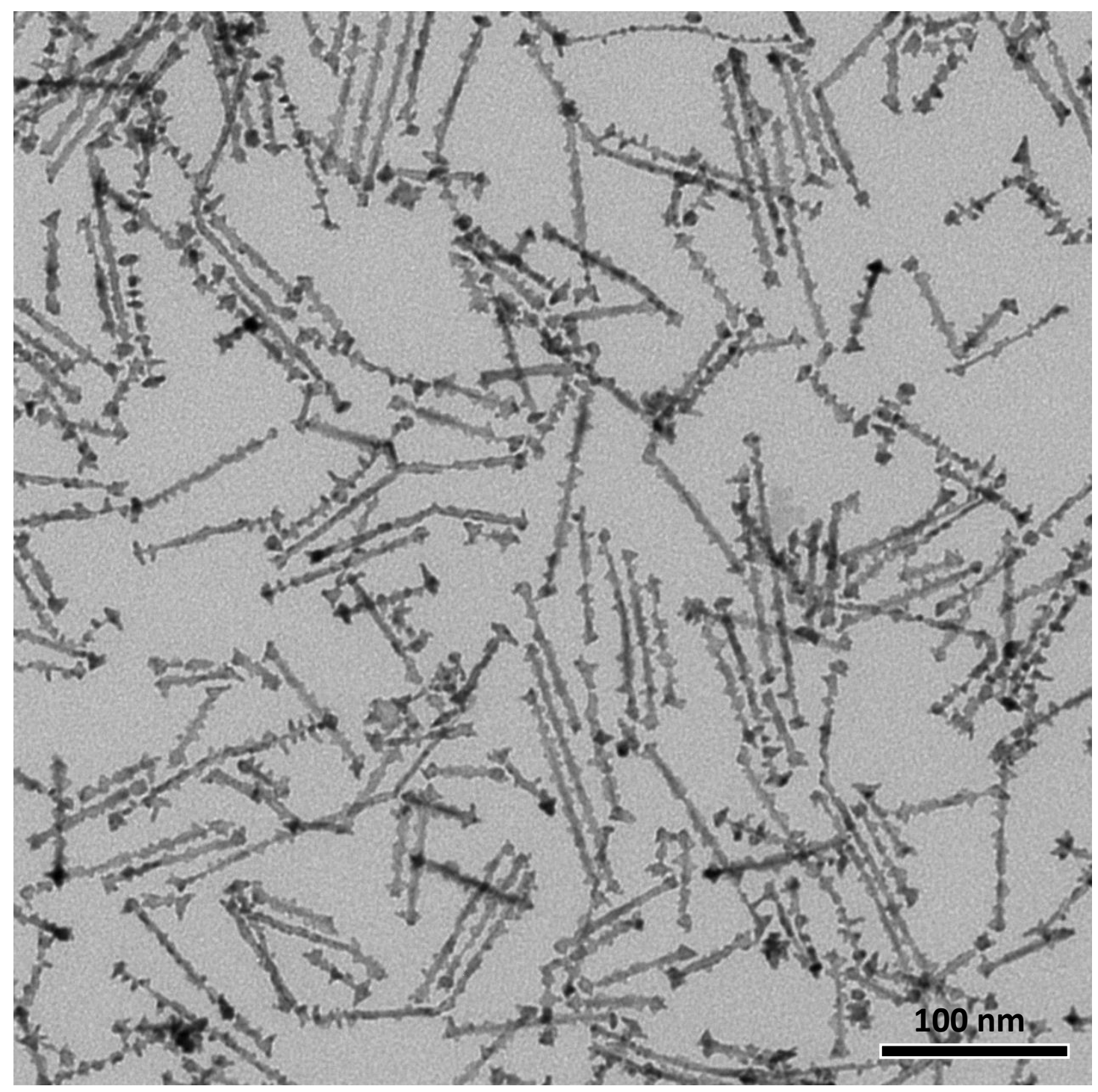

Figure S2. Additional TEM image of $\mathrm{Pt}_{5} \mathrm{FePd}_{2} \mathrm{NWs}$.
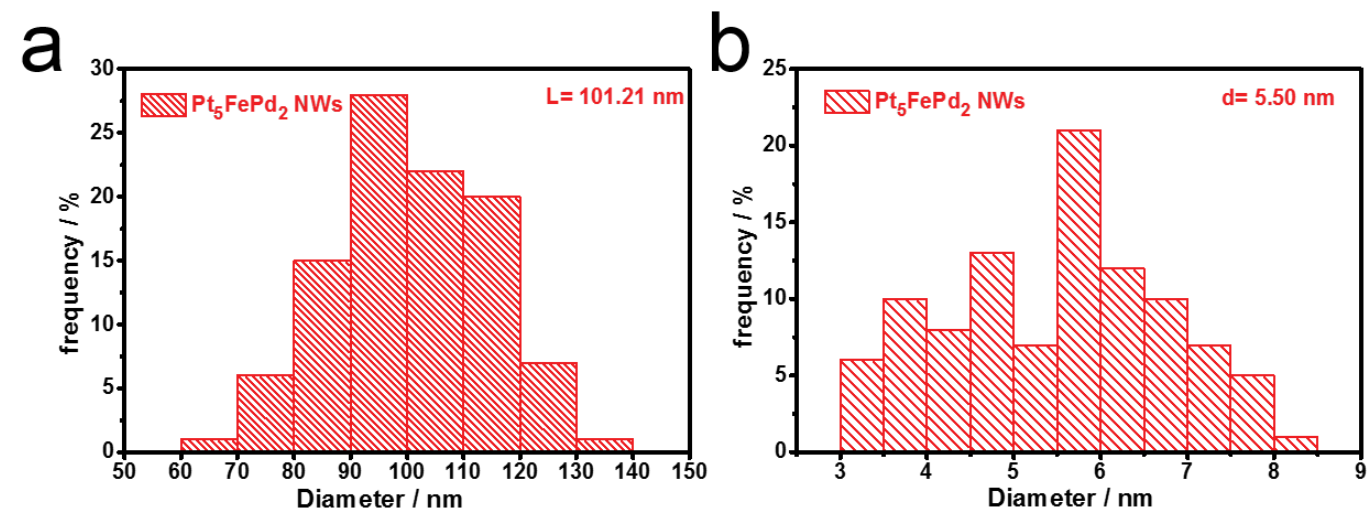

Figure S3. Size distribution of $\mathrm{Pt}_{5} \mathrm{FePd}_{2} \mathrm{NWs}$ (a) in length and (b) in width. 

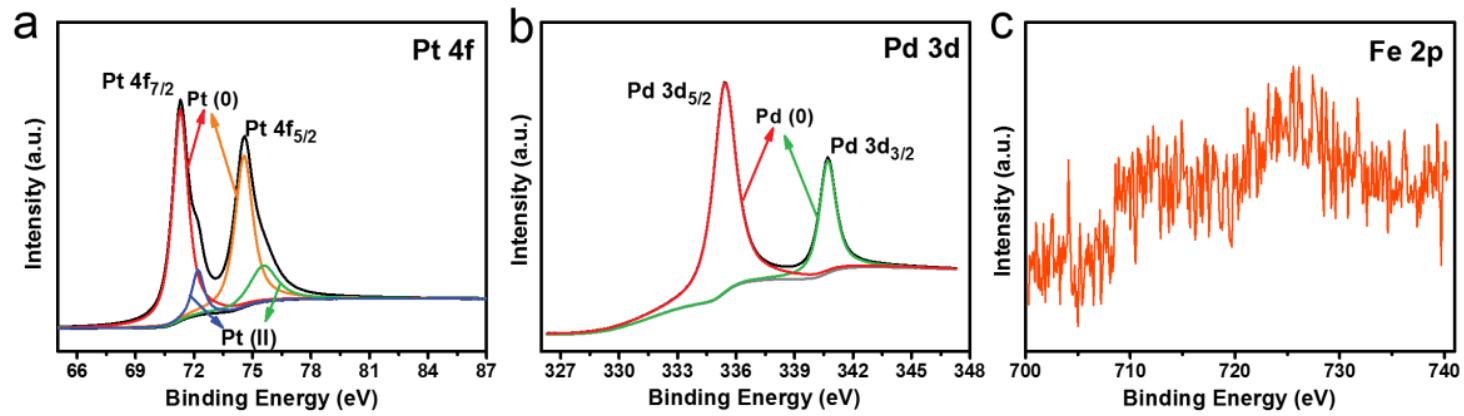

Figure S4. XPS spectra of (a)Pt 4f, (b) Pd 3d, (c) Fe 2p in $\mathrm{Pt}_{5} \mathrm{FePd}_{2} \mathrm{NWs}$.
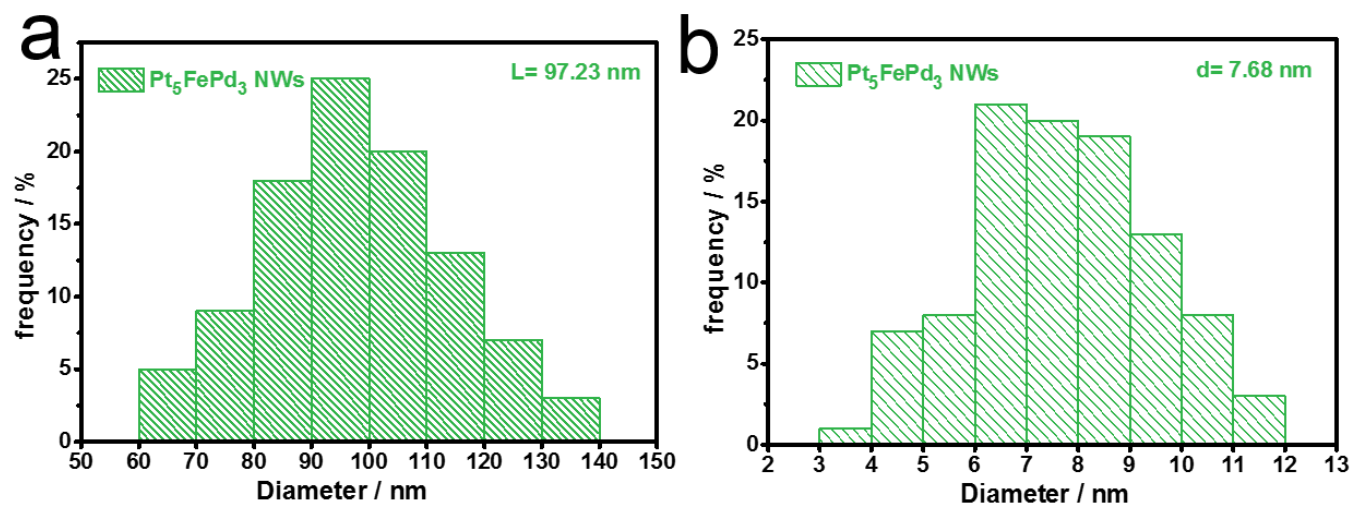

Figure S5. Size distribution of $\mathrm{Pt}_{5} \mathrm{FePd}_{3}$ NWs. (a) in length and (b) in width.
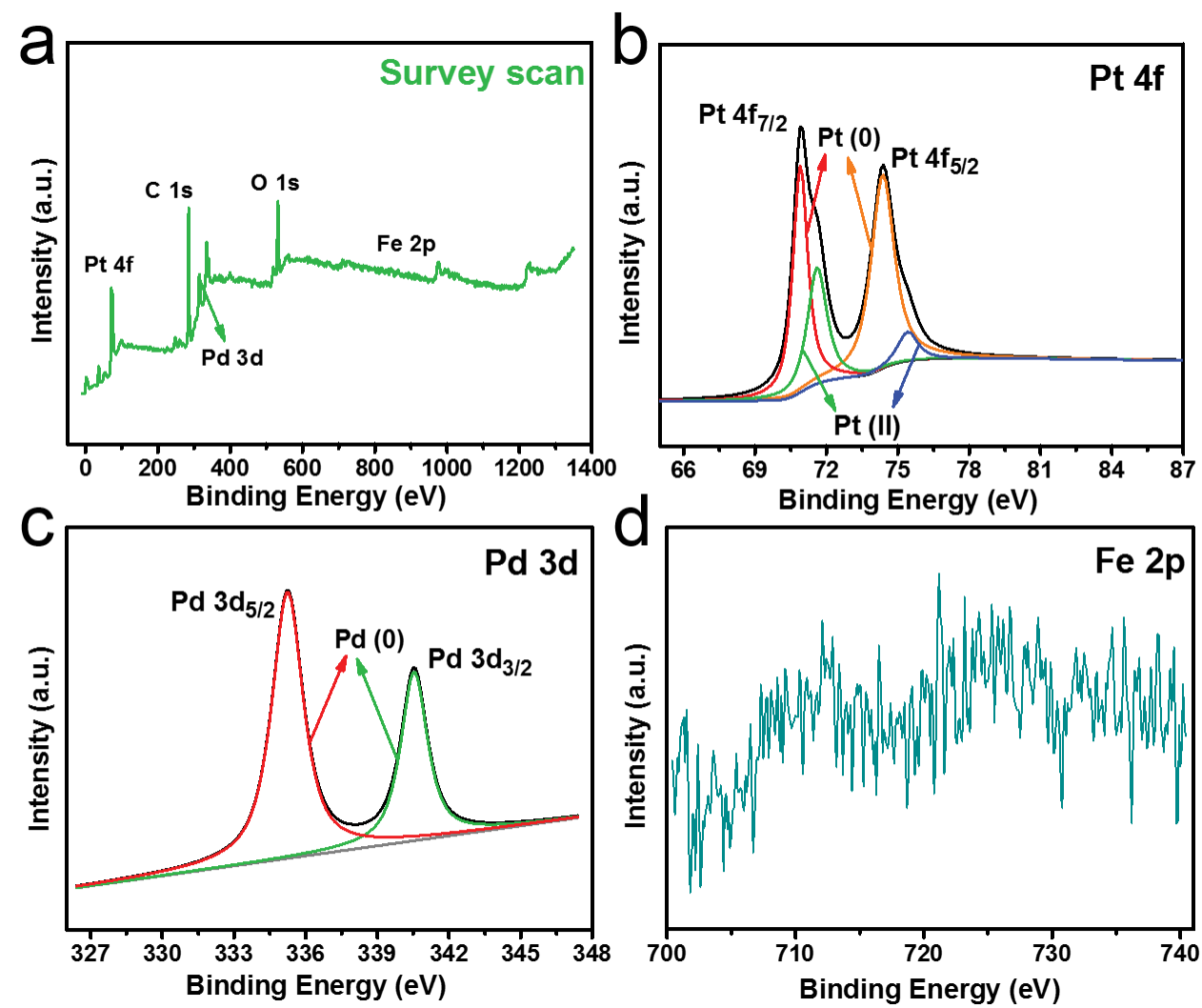
Figure S6. XPS spectra of (a) survey scan, (b) Pt 4f, (c) Pd 3d, and (d) Fe 2p in $\mathrm{Pt}_{5} \mathrm{FePd}_{3}$ NWs.
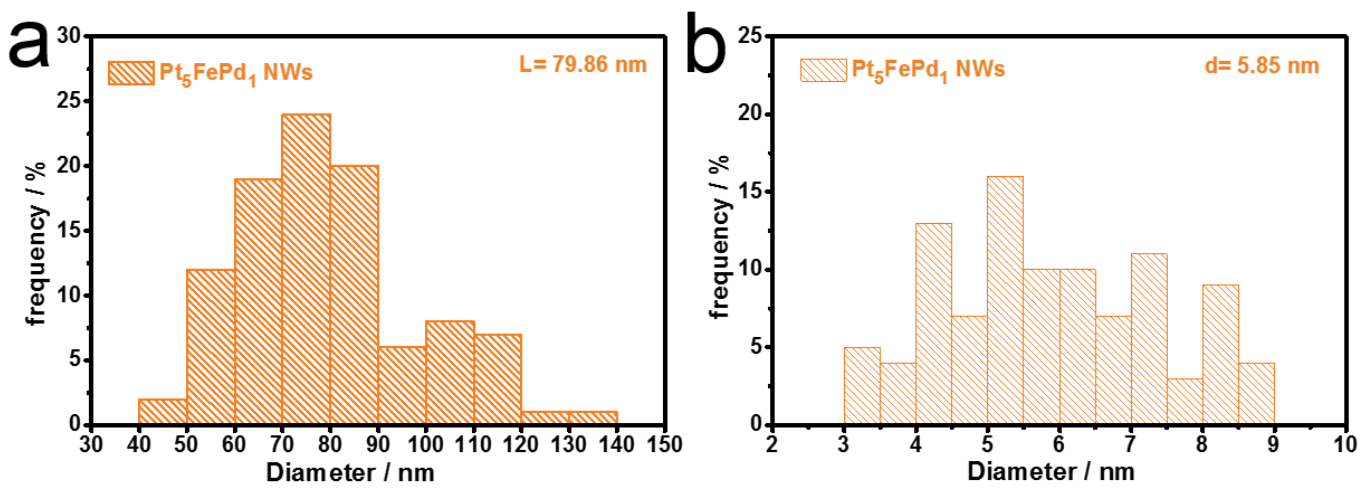

Figure S7. Size distribution of $\mathrm{Pt}_{5} \mathrm{FePd}_{1}$ NWs. (a) in length and (b) in width.

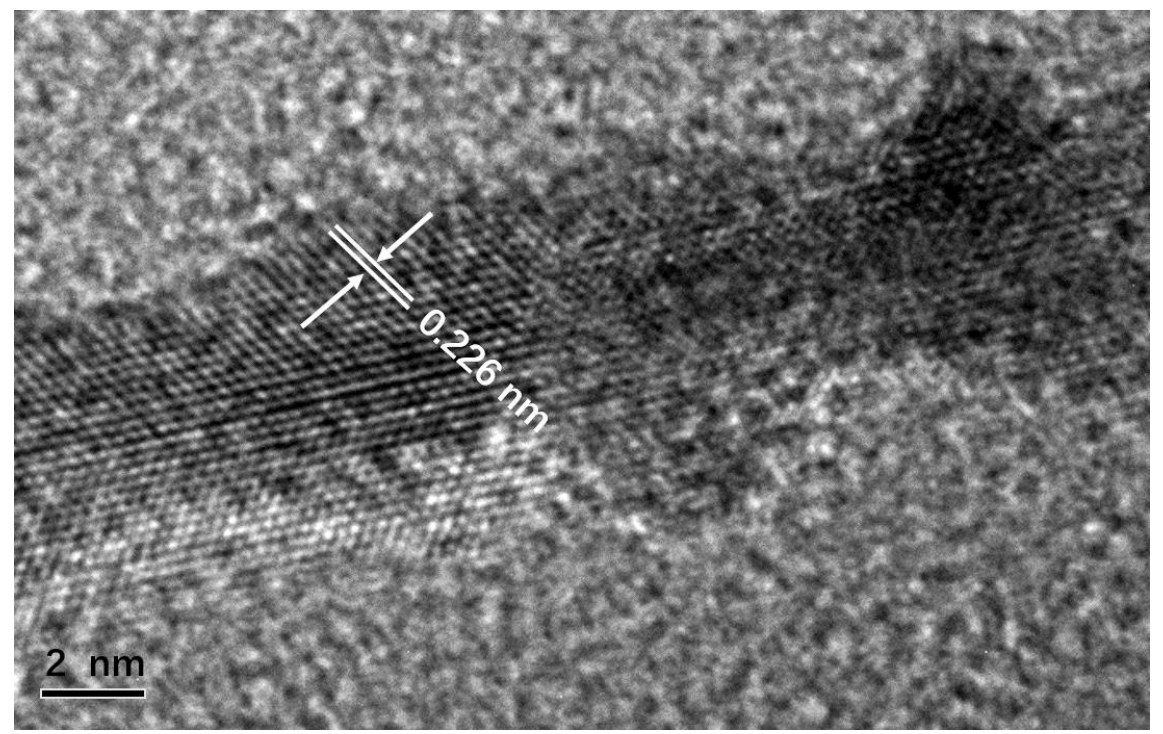

Figure S8. HRTEM image of $\mathrm{Pt}_{5} \mathrm{FePd}_{3}$ NWs. 

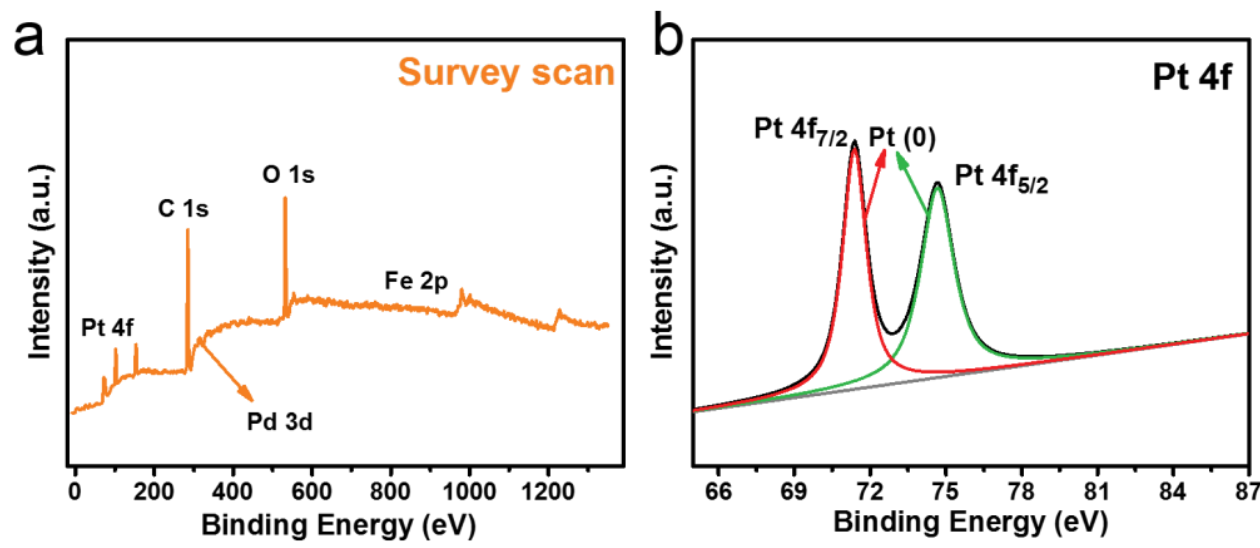

Figure S9. XPS spectra of (a) survey scan and (b) $\mathrm{Pt} 4 \mathrm{f}$ in $\mathrm{Pt}_{5} \mathrm{FePd}_{1} \mathrm{NWs}$.
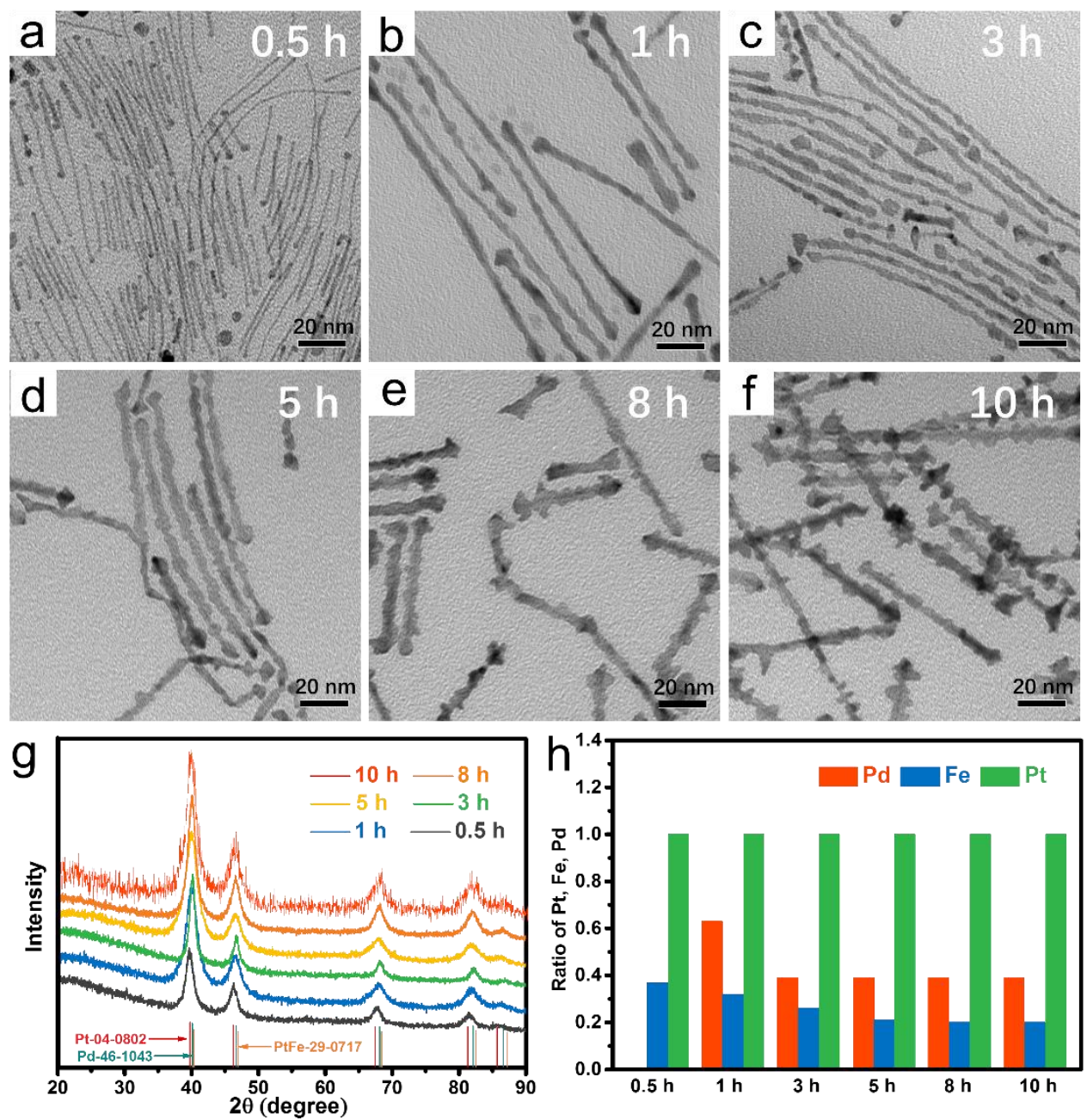

Figure S10. TEM images of $\mathrm{Pt}_{5} \mathrm{FePd}_{2} \mathrm{NWs}$ intermediates collected after the reaction had prolonged for (a) 0.5, (b) 1, (c) 3, (d) 5, (e) 8, and (f) $10 \mathrm{~h}$. (g) XRD patterns of $\mathrm{Pt}_{5} \mathrm{FePd}_{2} \mathrm{NWs}$ intermediates. (h) variation in the content of Pt, Fe and $\mathrm{Pd}$ in $\mathrm{Pt}_{5} \mathrm{FePd}_{2}$ 
NWs intermediates determined by SEM-EDS.
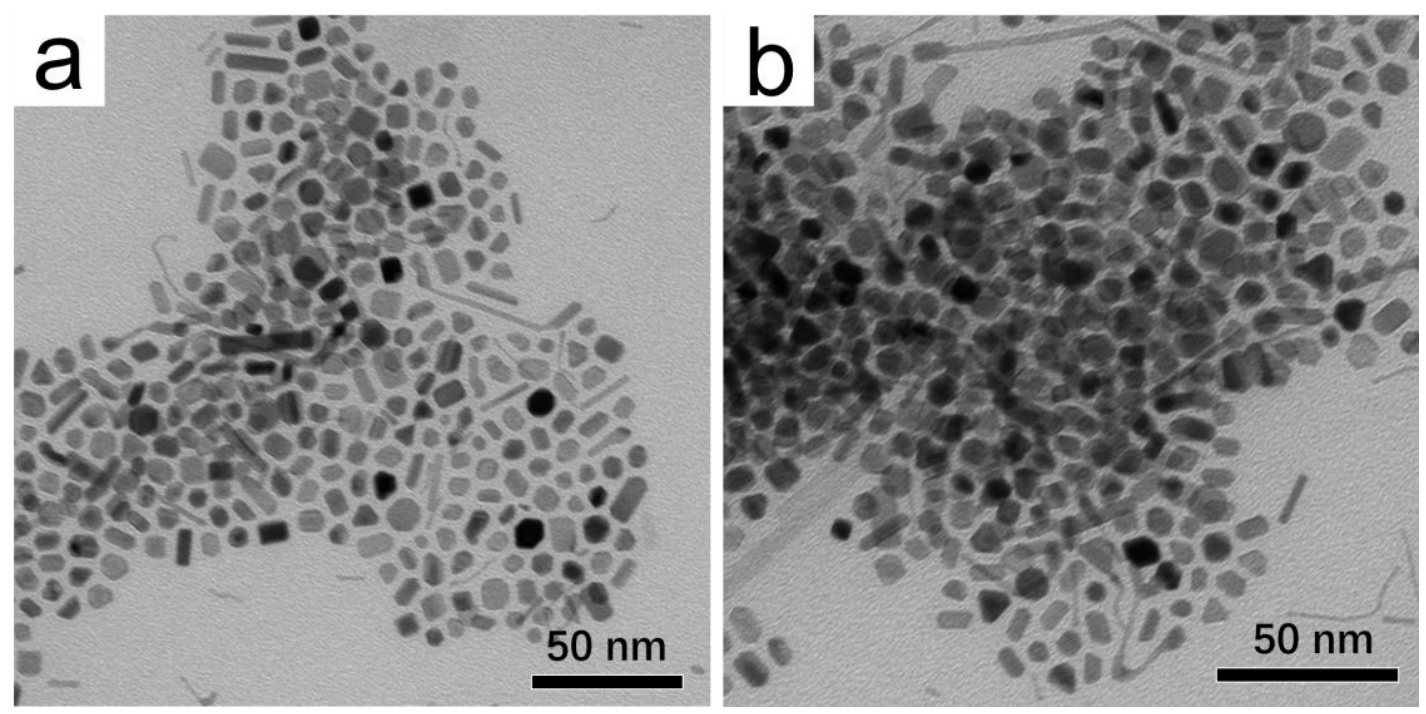

Figure S11. ( $a$ and b) TEM images of the products with the same reaction conditions as that of $\mathrm{Pt}_{5} \mathrm{FePd}_{2} \mathrm{NWs}$ without the addition of $\mathrm{Fe}(\mathrm{Ac})_{2}$. 

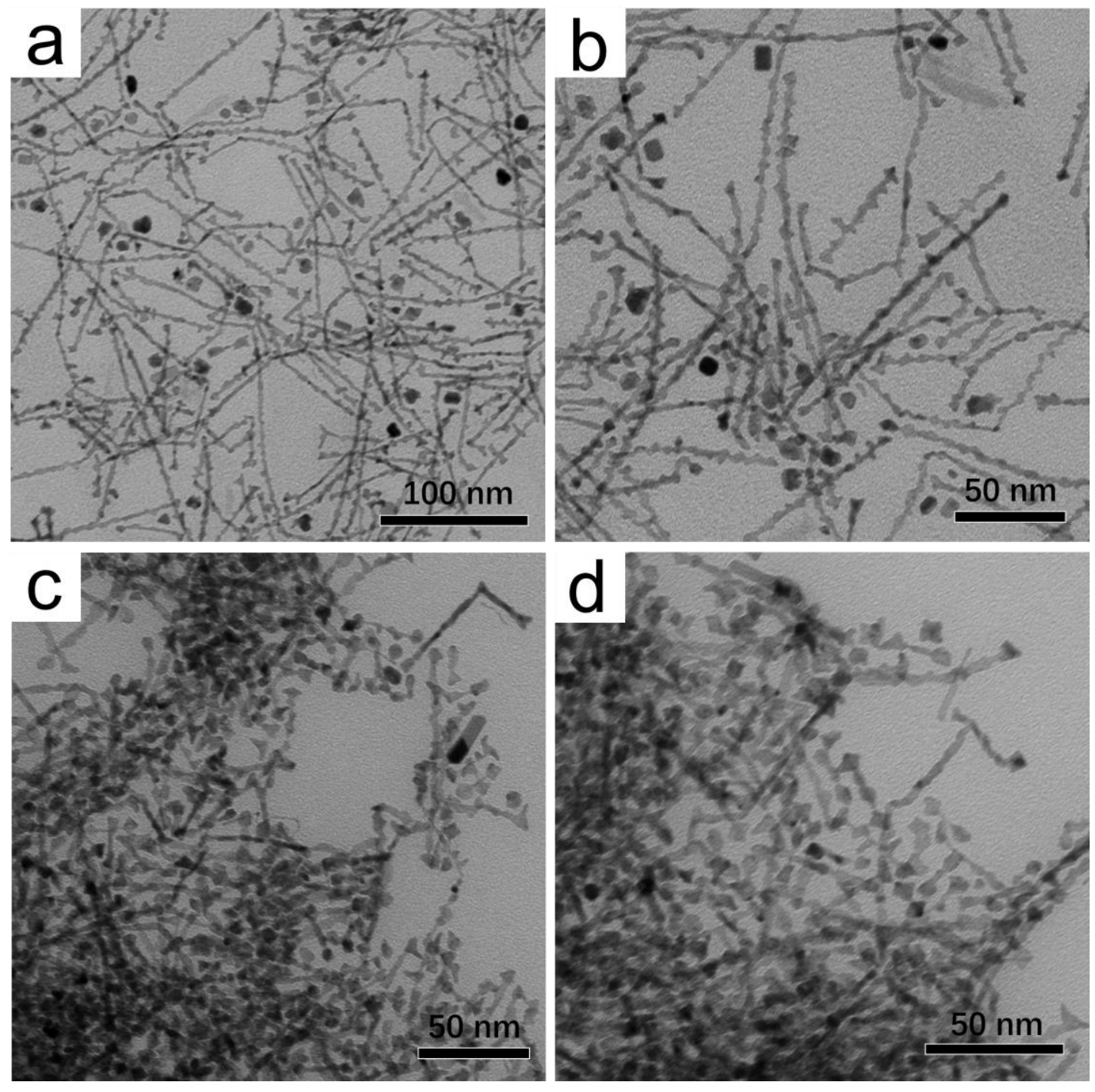

Figure S12. Representative TEM images of the products with the same reaction conditions as that of $\mathrm{Pt}_{5} \mathrm{FePd}_{2} \mathrm{NWs}$ without the addition of ( $\mathrm{a}$ and $\mathrm{b}$ ) glucose and ( $\mathrm{c}$ and d) phloroglucinol, respectively. 

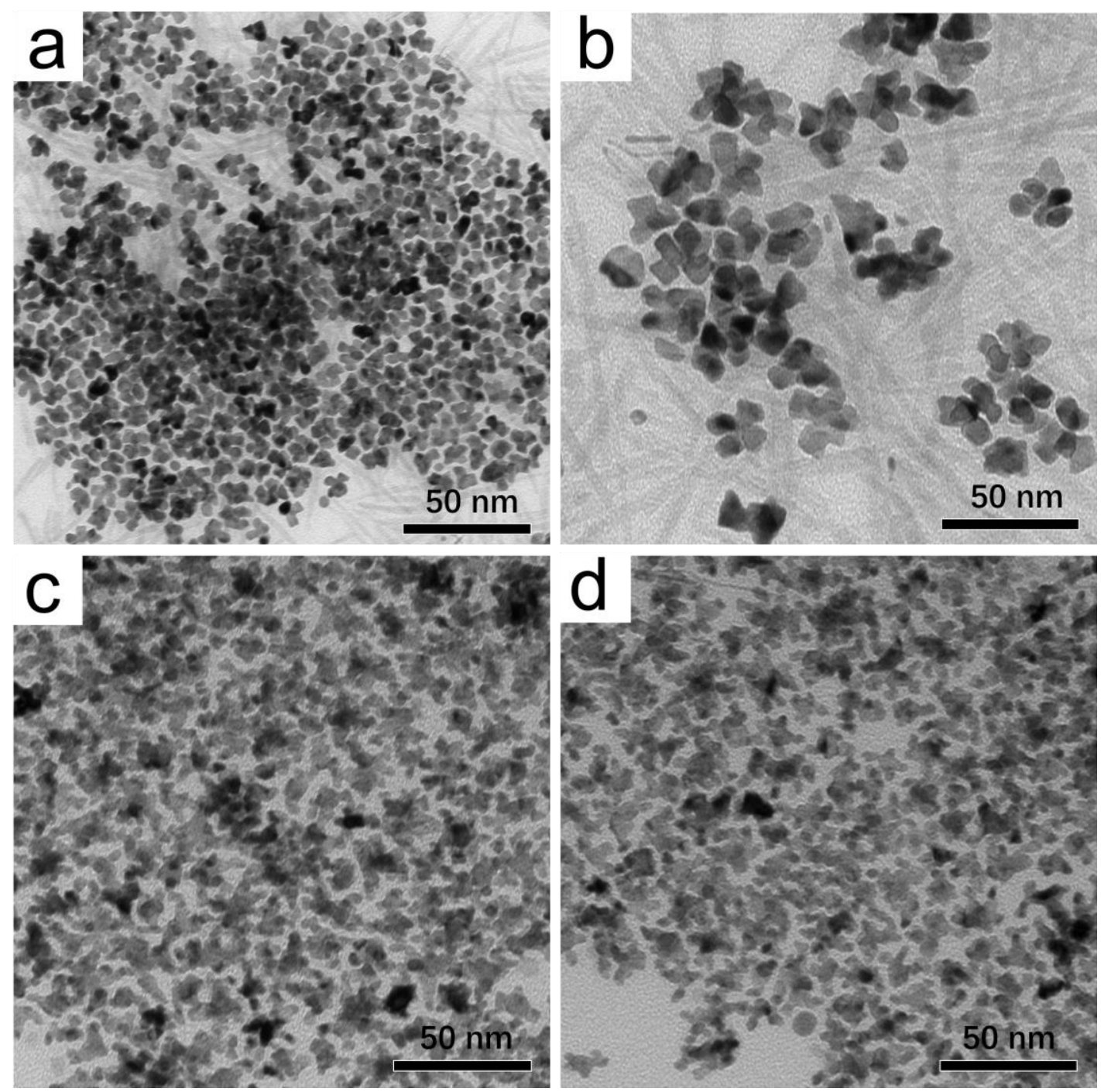

Figure S13. Representative TEM images of the products with the same reaction conditions as that of $\mathrm{Pt}_{5} \mathrm{FePd}_{2}$ NWs without the addition of (a and b) $\mathrm{W}(\mathrm{CO})_{6}$ and (c and d) CTAC, respectively.
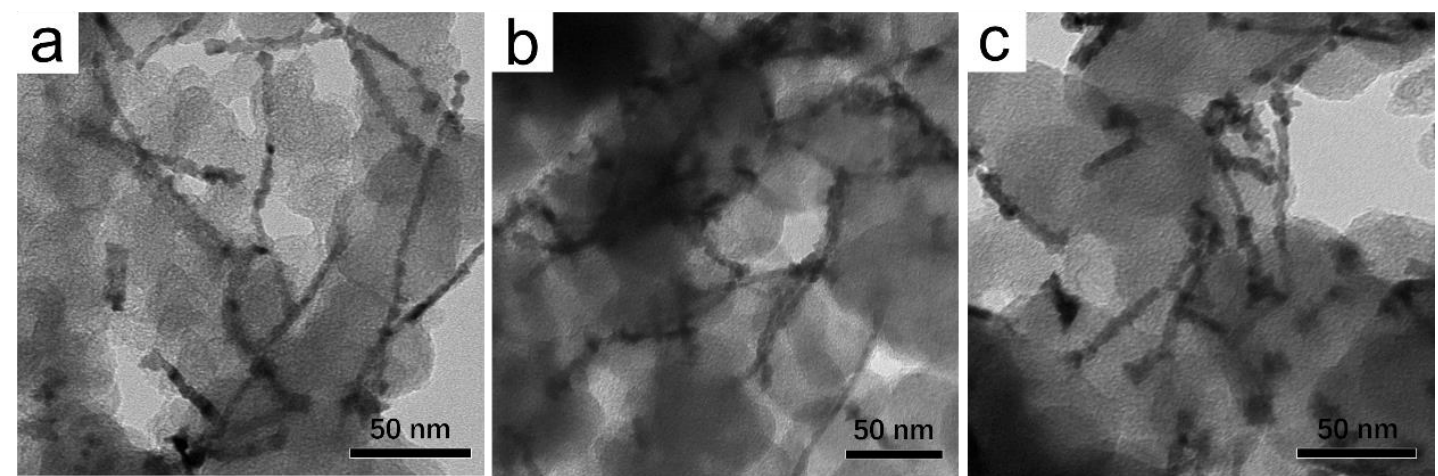

Figure S14. Representative TEM images of (a) $\mathrm{Pt}_{5} \mathrm{FePd}_{2} \mathrm{NWs}$, (b) $\mathrm{Pt}_{5} \mathrm{FePd}_{3} \mathrm{NWs}$, and 
(c) $\mathrm{Pt}_{5} \mathrm{FePd}_{1} \mathrm{NWs}$ catalysts before electrochemical measurements.

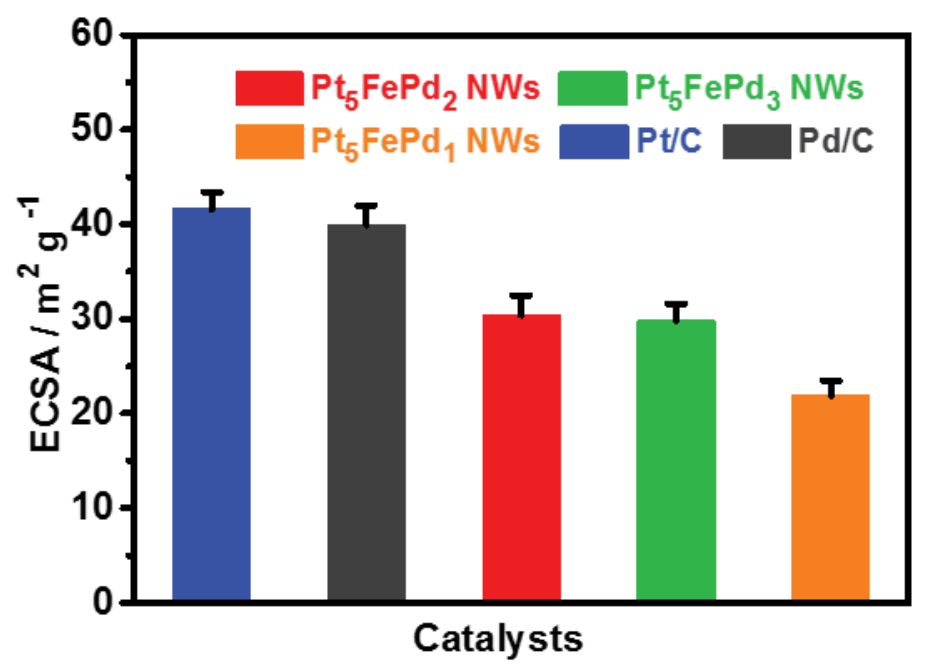

Figure S15. Calculated ECSA values of $\mathrm{Pt}_{5} \mathrm{FePd}_{2} \mathrm{NWs}, \mathrm{Pt}_{5} \mathrm{FePd}_{3} \mathrm{NWs}_{2} \mathrm{Pt}_{5} \mathrm{FePd}_{1} \mathrm{NWs}_{\text {, }}$ $\mathrm{Pt} / \mathrm{C}$, and $\mathrm{Pd} / \mathrm{C}$ catalysts.

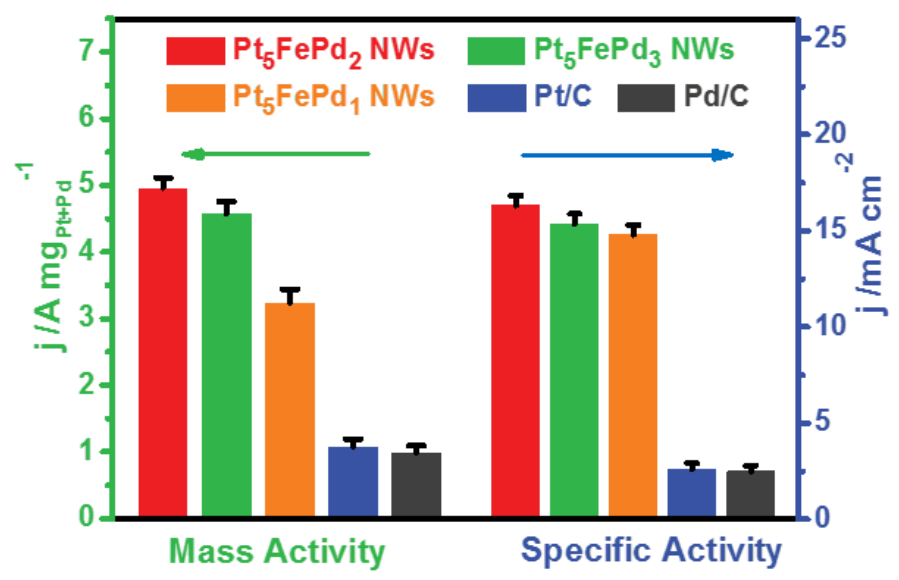

Figure S16. Mass activity and specific activity of $\mathrm{Pt}_{5} \mathrm{FePd}_{2} \mathrm{NWs}, \mathrm{Pt}_{5} \mathrm{FePd}_{3} \mathrm{NWs}$, $\mathrm{Pt}_{5} \mathrm{FePd}_{1} \mathrm{NWs}, \mathrm{Pt} / \mathrm{C}$, and $\mathrm{Pd} / \mathrm{C}$ catalysts towards EOR. 

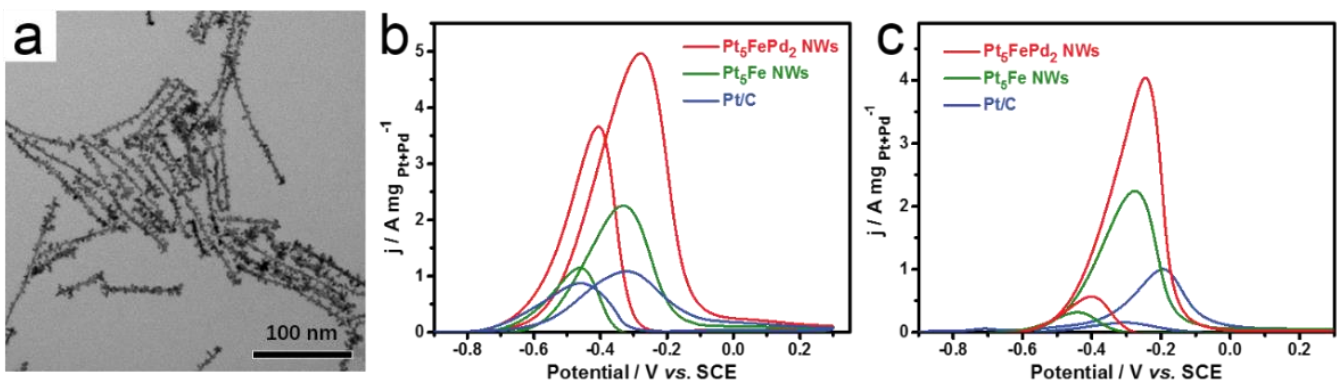

Figure S17. (a) TEM image of $\mathrm{Pt}_{5} \mathrm{Fe}$ NWs catalysts, $\mathrm{CV}$ curves of $\mathrm{Pt}_{5} \mathrm{FePd}_{2} \mathrm{NWs}, \mathrm{Pt}_{5} \mathrm{Fe}$ NWs, and Pt/C for (b) EOR and (c) MOR.
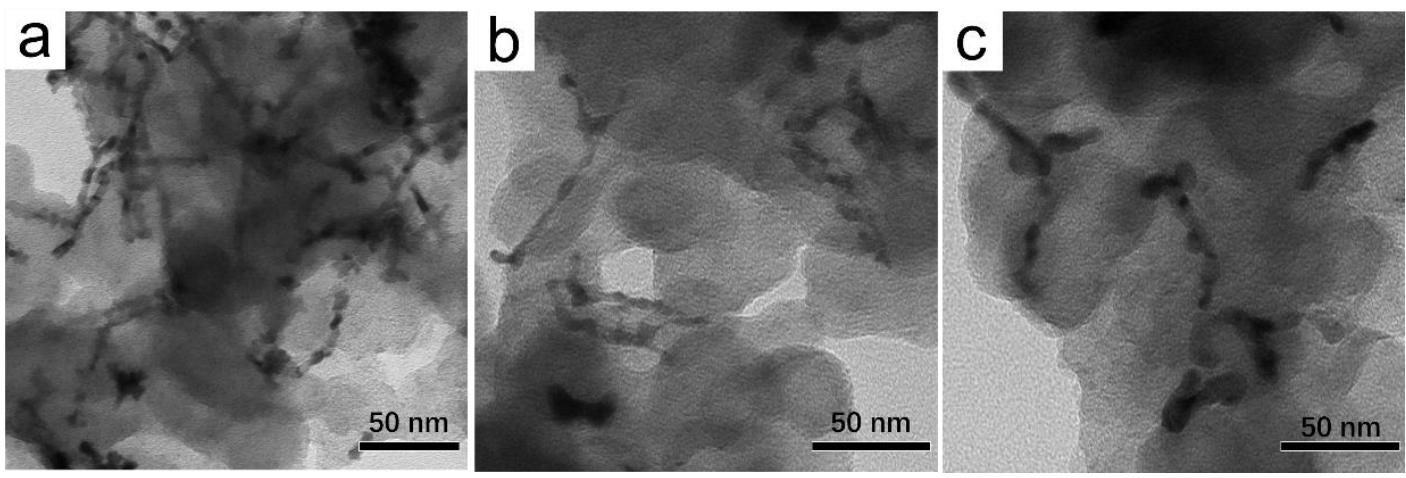

Figure S18. Representative TEM images of (a) $\mathrm{Pt}_{5} \mathrm{FePd}_{2} \mathrm{NWs}$, (b) $\mathrm{Pt}_{5} \mathrm{FePd}_{3} \mathrm{NWs}$, and (c) $\mathrm{Pt}_{5} \mathrm{FePd}_{1} \mathrm{NWs}$ catalysts after durability tests. 
Table S1. EOR performances of $\mathrm{Pt}_{5} \mathrm{FePd}_{2} \mathrm{NWs}$ and various electrocatalysts from published works.

\begin{tabular}{|c|c|c|c|c|}
\hline \multirow{2}{*}{ Catalysts } & \multicolumn{2}{|c|}{$\begin{array}{l}\text { Peak currents from CV } \\
\text { curves }\end{array}$} & \multirow{2}{*}{ Electrolyte } & \multirow{2}{*}{ Reference } \\
\hline & $\mathrm{J}_{\mathrm{m}}\left(\mathrm{A} \mathrm{mg}^{-1}\right)$ & $\mathrm{J}_{\mathrm{s}}\left(\mathrm{mA} \mathrm{cm}{ }^{-2}\right)$ & & \\
\hline $\begin{array}{c}\mathrm{Pt}_{5} \mathrm{FePd}_{2} \\
\mathrm{NWs}\end{array}$ & 4.965 & 16.33 & $\begin{array}{c}1 \mathrm{M} \mathrm{KOH}+1 \mathrm{M} \\
\text { ethanol }\end{array}$ & This work \\
\hline PtPd CANs & 1.075 & & $\begin{array}{c}0.5 \mathrm{M} \mathrm{KOH}+1 \\
\text { M ethanol }\end{array}$ & $\begin{array}{l}\text { J. Mater. Chem. A, 2014, 2, 13840- } \\
13844 .\end{array}$ \\
\hline $\begin{array}{c}\mathrm{Ni} @ \mathrm{Au} @ \mathrm{Pd} / \\
\mathrm{rGO}\end{array}$ & & 8.85 & $\begin{array}{c}1 \mathrm{M} \mathrm{KOH}+1 \mathrm{M} \\
\text { ethanol }\end{array}$ & Electrochim. Acta. 2018, 271, 1-9. \\
\hline $\mathrm{Pt}_{1} \mathrm{Mo}_{1} / \mathrm{C}$ & 1.0 & & $\begin{array}{c}1 \mathrm{M} \mathrm{KOH}+0.5 \\
\text { M ethanol }\end{array}$ & $\begin{array}{l}\text { Appl. Catal. B-Environ. 2017, 203, } \\
654-662 .\end{array}$ \\
\hline $\mathrm{PtPd} / \mathrm{RGO}$ & 1.487 & & $\begin{array}{c}1 \mathrm{M} \mathrm{KOH}+1 \mathrm{M} \\
\text { ethanol }\end{array}$ & $\begin{array}{l}\text { ACS Appl. Mater. Interfaces 2014, } 6 \text {, } \\
\text { 3607-3614. }\end{array}$ \\
\hline PdRu & & 12.3 & $\begin{array}{c}1 \mathrm{M} \mathrm{KOH}+1 \mathrm{M} \\
\text { ethanol }\end{array}$ & Nanoscale, 2018, 10, 12605-12611 \\
\hline $\mathrm{Pt}_{68} \mathrm{Cu}_{32}$ & 2.33 & & $\begin{array}{l}0.5 \mathrm{M} \mathrm{KOH}+ \\
0.5 \mathrm{M} \text { ethanol }\end{array}$ & Nanoscale, 2017, 9, 2963-2968 \\
\hline $\mathrm{PtAg}_{2} / \mathrm{C}-\mathrm{D}$ & 2.426 & & $\begin{array}{c}1 \mathrm{M} \mathrm{KOH}+1 \mathrm{M} \\
\text { ethanol }\end{array}$ & Electrochim. Acta 2017, 236, 72-81 \\
\hline
\end{tabular}


Table S2. MOR performances of $\mathrm{Pt}_{5} \mathrm{FePd}_{2} \mathrm{NWs}$ and various electrocatalysts from published works.

\begin{tabular}{|c|c|c|c|c|}
\hline \multirow{2}{*}{ Catalysts } & \multicolumn{2}{|c|}{$\begin{array}{l}\text { Peak currents from CV } \\
\text { curves }\end{array}$} & \multirow{2}{*}{ Electrolyte } & \multirow{2}{*}{ Reference } \\
\hline & $\mathrm{J}_{\mathrm{m}}\left(\mathrm{A} \mathrm{mg}^{-1}\right)$ & $\mathrm{J}_{\mathrm{s}}\left(\mathrm{mA} \mathrm{cm}{ }^{-2}\right)$ & & \\
\hline $\begin{array}{c}\mathrm{Pt}_{5} \mathrm{FePd}_{2} \\
\mathrm{NWs}\end{array}$ & 4.038 & 13.28 & $\begin{array}{c}1 \mathrm{M} \mathrm{KOH}+1 \mathrm{M} \\
\text { methanol }\end{array}$ & This work \\
\hline PtPdPt & 0.706 & & $\begin{array}{l}1 \mathrm{M} \mathrm{NaOH}+1 \\
\mathrm{M} \text { methanol }\end{array}$ & $\begin{array}{l}\text { Electrochim. Acta. 2016, 192, 400- } \\
406\end{array}$ \\
\hline PdRu & & 4.5 & $\begin{array}{c}1 \mathrm{M} \mathrm{NaOH}+1 \\
\text { M methanol }\end{array}$ & Nanoscale, 2018, 10, 12605-12611 \\
\hline $\mathrm{Pd}_{3} \mathrm{Ru} / \mathrm{C}$ & 1.037 & & $\begin{array}{l}0.5 \mathrm{M} \mathrm{KOH} \mathrm{+} \\
0.5 \mathrm{M} \text { methanol }\end{array}$ & J. Power Sources 2016, 303,182-193. \\
\hline $\mathrm{Ni@Pt}$ & 0.216 & & $\begin{array}{c}0.5 \mathrm{M} \mathrm{KOH} \text { and } \\
0.2 \mathrm{M} \mathrm{CH}_{3} \mathrm{OH}\end{array}$ & $\begin{array}{l}\text { J. Electroanal. Chem. 2017, 795, } 17- \\
25\end{array}$ \\
\hline $\mathrm{Pd}-\mathrm{Cu}$ & 0.659 & & $\begin{array}{c}1 \mathrm{M} \mathrm{KOH}+0.5 \\
\text { M methanol }\end{array}$ & $\begin{array}{l}\text { Appl. Catal. A: General 2015, 492, } \\
\text { 100-106. }\end{array}$ \\
\hline $\mathrm{Pt} @ \mathrm{HfSx} / \mathrm{CNT}$ & & 4.98 & $\begin{array}{c}1 \mathrm{M} \mathrm{KOH}+0.1 \\
\text { M methanol }\end{array}$ & $\begin{array}{l}\text { J. Power Sources 2019, 410-411, } \\
\text { 204-212. }\end{array}$ \\
\hline $\mathrm{Pt}-\mathrm{Bi} / \mathrm{GNs}$ & 2.005 & & $\begin{array}{c}1 \mathrm{M} \mathrm{KOH}+1 \mathrm{M} \\
\text { methanol }\end{array}$ & Electrochim. Acta. 2018, 264, 53-60 \\
\hline
\end{tabular}

